\title{
Thorough Mastication Prior to Swallowing Increases Postprandial Satiety and the Thermic Effect of a Meal in Young Women
}

\author{
Naho Komai ${ }^{1}$, Naoko MotoKuBotA ${ }^{1}$, Maki SuzUKI ${ }^{1}$, Ikuyo HAYASHI ${ }^{1}$, \\ Toshio MORITANI ${ }^{2}$ and Narumi NAGAI ${ }^{1, *}$ \\ ${ }^{1}$ Graduate School of Human Science and Environment, University of Hyogo, \\ 1-1-12 Shinzaike-honcho, Himeji, Hyogo 670-0092, Japan \\ ${ }^{2}$ Graduate School of Human and Environmental Studies, Kyoto University, \\ Sakyo-ku, Kyoto 606-8501, Japan \\ (Received January 7, 2016)
}

\begin{abstract}
Summary There is evidence to support that mastication may contribute to the prevention of weight gain via reduction of appetite sensations and subsequent energy intake. However, the metabolic effect of mastication after consumption of a daily meal, composed of the staple food (rice), soup, main and side dishes, is limited. Therefore, the effect of thorough mastication on greater satiety and the thermic effect of a meal (TEM) was investigated in young women. In study 1, energy expenditure (EE) derived from masticatory muscle activity for 20 min was measured while chewing hard, tasteless, non-caloric gum in seven subjects. In study 2 , ten subjects consumed a solid meal performing 30 chews per mouthful (30 CPM), or swallowed the same, pureed meal without chewing (0 CPM) on two separate days, and postprandial EE, substrate oxidation, subjective appetite ratings and autonomic nervous system (ANS) activity for $3 \mathrm{~h}$ were examined. Both test meals were iso-caloric $(2,510 \mathrm{~kJ})$ and -weighted $(884 \mathrm{~g})$, and consumed in $20 \mathrm{~min}$. From study 1, the EE of mastication itself for the $20 \mathrm{~min}$ was estimated to be $3.7 \pm 0.8 \mathrm{~kJ}$. From study 2 , significantly higher TEM $(134.2 \pm 15.5$ vs. $67.8 \pm 13.8 \mathrm{~kJ} / 3 \mathrm{~h}, p<0.001)$ as well as satiety $(p=0.005)$, and tendency toward greater fat oxidation $(p=0.090)$ and ANS activity $(p=0.069)$ were observed after consumption of the meal with $30 \mathrm{CPM}$ compared to $0 \mathrm{CPM}$. In conclusion, thorough mastication before swallowing increased postprandial satiety and the TEM in young women, suggesting such eating behavior may be useful for preventing obesity.
\end{abstract}

Key Words mastication, masticatory muscle, satiety, thermic effect of a meal, daily meal

Energy-dense, highly-processed foods that require little effort in mastication may promote excessive energy intake (1). Some reports (2-5) demonstrated that a reduced feeling of satiety together with lower postprandial thermogenesis was observed in healthy subjects following direct introduction of a meal into the stomach via a tube in comparison to a meal equivalent in amount and calories consumed normally.

This phenomenon has been investigated in animal studies $(6,7)$, demonstrating the association of mastication with postprandial satiety and increased thermogenesis through activation of histamine neurons. Additionally, histamine neurons are involved in lipolysis induced by the uncoupling of protein-1 via increase in sympathetic efferent nerve activity, suggesting that mastication may have a preventive effect on weight gain (7). Indeed, soft pellet-fed rats showed greater adiposity together with lower postprandial thermogenesis than normal pellet-fed rats (8).

Generally, oral processing, including smell, taste, and the physical sensation of food, sends signals to the satiety center of the brain (9); however, these signals may

\footnotetext{
*To whom correspondence should be addressed.

E-mail: nagai@shse.u-hyogo.ac.jp
}

be reduced when an iso-caloric meal is consumed more quickly. Recent studies have suggested that eating slowly (10) or increasing chewing before swallowing $(11,12)$ may induce higher postprandial satiety as well as a greater response to anorexigenic gut hormones. These results raise the possibility that increasing mastication may be a simple and beneficial behavior for the prevention of weight gain. However, few human studies have evaluated the effect of increased mastication on postprandial thermogenesis when consuming daily meals composed of the staple food (rice), soup, main and side dishes.

Accordingly, we hypothesized that thorough mastication may induce a greater feeling of satiety and postprandial energy expenditure (EE) compared to swallowing without mastication. Based on the hypothesis, we designed two studies, 1) to estimate EE derived from masticatory muscle activity, and 2) to evaluate the effect of mastication on postprandial satiety and the thermic effect of a meal (TEM) after an iso-caloric solid or pureed meal in young female volunteers.

\section{MATERIALS AND METHODS}

Subjects. We recruited seventeen female university students from our campus for the present study. All sub- 
Table 1. Nutrient composition of the test meal. ${ }^{1}$

\begin{tabular}{lcccrrr}
\hline & Weight $(\mathrm{g})$ & Energy $(\mathrm{kJ})$ & Protein $(\mathrm{g})$ & Fat $(\mathrm{g})$ & Carb $(\mathrm{g})$ & Na $(\mathrm{mg})$ \\
\hline Commercial set meal $^{2}$ & 300 & 1,377 & 16.1 & 15.8 & 30.5 & 904 \\
Boiled rice $_{\text {Soup }^{3}}$ & 165 & 1,013 & 3.4 & 0 & 56.0 & 0 \\
Water $^{4}$ & 169 & 134 & 2.2 & 1.2 & 3.2 & 719 \\
Total & 250 & & & & \\
& 884 & 2,524 & 21.7 & 17.0 & 89.7 & 1,623 \\
\hline
\end{tabular}

${ }^{1}$ The macronutrient composition and sodium of the meals were calculated using computer-assisted procedures based on the Japanese Food Composition Table. Sodium chloride equivalent was $4.1 \mathrm{~g}$.

${ }^{2}$ Hamburger patty with mashed potato, omelet, and boiled vegetables.

${ }^{3}$ Freeze-dried miso-flavored soup made with $160 \mathrm{~mL}$ hot water.

${ }^{4}$ Drinking water with test meal.

jects were non-smokers and non-obese, not habitually skipping any meals, not suffering from food allergies or food intolerances, and free of any medical or dental symptoms that affect mastication or energy metabolism. The SHSE (School of Human Science and Environment) Research Ethics Committee of the University of Hyogo approved the study (No. 116), which was in accordance with the principles of the Declaration of Helsinki. All subjects provided written informed consent.

Experimental procedures. All subjects were asked to maintain their usual lifestyle and body mass for at least 1 mo before the test. On the day before the test, the consumption of coffee, tea, spicy foods, and high-fat foods was prohibited, and no sports activities were permitted that evening. On the morning of the test, each subject came to our laboratory at 8 a.m. after an overnight fast beginning at 10 p.m. the previous night. After subjects changed into examination clothes, body mass and body fat percentage were measured using a bioelectrical impedance analyzer (InBody520, Biospace Co., Seoul, Korea), and seated blood pressure was measured twice using an electrical sphygmomanometer (HEM-7250-IT, OMRON Co., Ltd., Kyoto, Japan) on the first visit for studies 1 and 2 .

Study 1: EE derived from masticatory muscle activity: To estimate EE derived from masticatory muscle activity, EEs were measured before and after masticating a tasteless, hard, non-caloric gum (1.0 g, CAT 21, Willdent Ltd., Osaka, Japan) for $20 \mathrm{~min}$ in 7 subjects (mean \pm SE, age, $21.6 \pm 0.6 \mathrm{y} ; \mathrm{BMI}, 18.5 \pm 0.7 \mathrm{~kg} / \mathrm{m}^{2}$ ) under the assumption that the difference in EEs before and after mastication would represent the EE of mastication itself (masticatory muscle activity) in the fasting state. The subjects were instructed to keep chewing with their normal chewing frequency.

Subjects were tested in the morning, from 8 to 9 a.m., after fasting. After anthropometric measurements, subjects were prepared for an electrocardiogram (ECG) and then rested for at least $20 \mathrm{~min}$ in a semirecumbent position in a temperature-controlled $\left(24-25^{\circ} \mathrm{C}\right)$ room. After adjustment of the mask, first ECG and gas exchange parameters (GAS) were continuously recorded for 15 min using an open-circuit computerized indirect calorimeter used in our previous study (13) (Oxygen Analyzer FC-10, Carbon Dioxide Analyzer CA-10, Sable Systems, Berlin, Germany; Air Flow Sensor BAF-201, Biotech, Kyoto, Japan). The calorimeter was calibrated before each test with a reference gas mixture $\left(15 \% \mathrm{O}_{2}\right.$ and $5 \% \mathrm{CO}_{2}$ ). Continuous ventilatory volumes, oxygen consumption and carbon dioxide generation were averaged for each 15-s period, and the mean value for each minute was calculated and printed out. The respiratory quotient was determined utilizing oxygen consumption and carbon dioxide generation. The resting EE was calculated according to the method outlined by Lusk (14). Immediately after the 20-min chewing, the gum was removed from the mouth, and the same measurements were repeated. The EE of mastication itself was calculated as the difference in EEs before and after chewing.

Detailed information on the quantification of the autonomic nervous system (ANS) activity by means of heart rate variability power spectral analysis has been described in previous studies $(13,15)$.

To assess subjective appetite ratings, the subjects rated their hunger, fullness, satiety and prospective food intake using visual analogue scales (VAS) $(16,17)$ before and after the 20-min mastication period. The VAS were $100 \mathrm{~mm}$ long horizontal lines with words expressing the most negative and most positive rating anchored at each end. The questionnaires were made as small booklets showing one question at a time. The subjects were asked to make a univocal mark on the horizontal line that corresponded to their feelings $(16,17)$.

Study 2: The masticatory efficacy on TEM and appetite sensations

Experimental protocol: Ten subjects (mean \pm SE, age, $20.6 \pm 0.6 \mathrm{y}$; BMI, $20.0 \pm 0.4 \mathrm{~kg} / \mathrm{m}^{2}$ ) visited our laboratory twice with an interval of 1 to $11 \mathrm{~d}$. Anthropometric and preprandial measurements, including appetite ratings, ECG and GAS, were measured as described in study 1. For the postprandial period, ECG and GAS samples were taken for 6-min periods every $30 \mathrm{~min}$ (24min interval) over a 3-h period. During the test period, subjects remained seated quietly in bed either reading a book or using their smart phone. The postprandial EE, carbohydrate oxidation, and fat oxidation were calcu- 


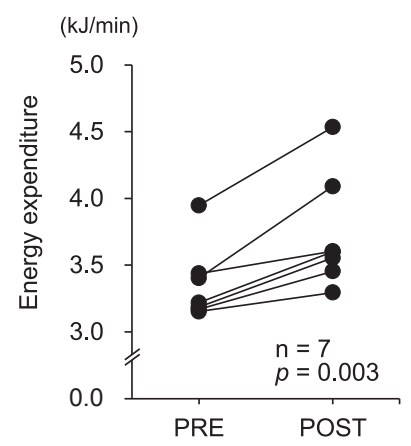

Fig. 1. Individual changes in EE at baseline (PRE) and after 20 min of chewing a tasteless, hard, non-caloric gum (POST). $p$ value was analyzed by paired $t$-test (PRE vs. POST).

lated as the area under the curve (AUC) using the trapezoidal rule. The 3-h TEM was calculated as net increase above preprandial EE. Appetite was measured immediately after ingestion and every 30 min over the following 3-h period (0, 30, 60, 90, 120, 150 and $180 \mathrm{~min})$.

Test meals: Subjects consumed a solid meal with 30 chews per mouthful (30 CPM) or swallowed a pureed meal without chewing (O CPM) on 2 different days in a randomized order. Each test meal was iso-caloric $(2,524 \mathrm{~kJ}, 60 \%$ of energy as carbohydrate, $15 \%$ of energy as protein, and $25 \%$ of energy as fat) and -weighted $(884 \mathrm{~g})$, which corresponded to about one third of the subject's daily estimated energy requirements, in accordance with the reference dietary intake for Japanese (18), and was consumed in $20 \mathrm{~min}$. The test meals were composed of ordinary Japanese breakfast foods, and were prepared in the kitchen adjacent to our laboratory. All ingredients of the test meal are presented in Table 1. The solid meal was served on three plates and served with chopsticks. The pureed meal was made from the same solid meal. The rice and soup were placed in a mixer and blended into a homogeneous puree, and the remaining dishes were made into another homogeneous puree with hot water. All test meals were served in a warm state.

Statistical analyses. The data were expressed as the means \pm SE. In study 1 , comparison of the pre- and postprandial EE was analyzed by Student's paired $t$ - test. In study 2, the time course of VAS, EE, fat and carbohydrate oxidation, and ANS parameters was analyzed by two factor (group $\times$ time) repeated ANOVA. Statistical comparison of the metabolic parameter AUCs between 30 CPM and O CPM was performed by Student's paired $t$-test. All analyses were performed using SPSS ver. 20 for Windows (IBM Inc., Tokyo, Japan). $p<0.05$ was considered statistically significant.

\section{RESULTS}

\section{Study 1}

EE derived from masticatory muscle activity. Individual changes in EE before and after the 20-min mastication are shown in Fig. 1. After the mastication period, a significant increase in EE was observed com-

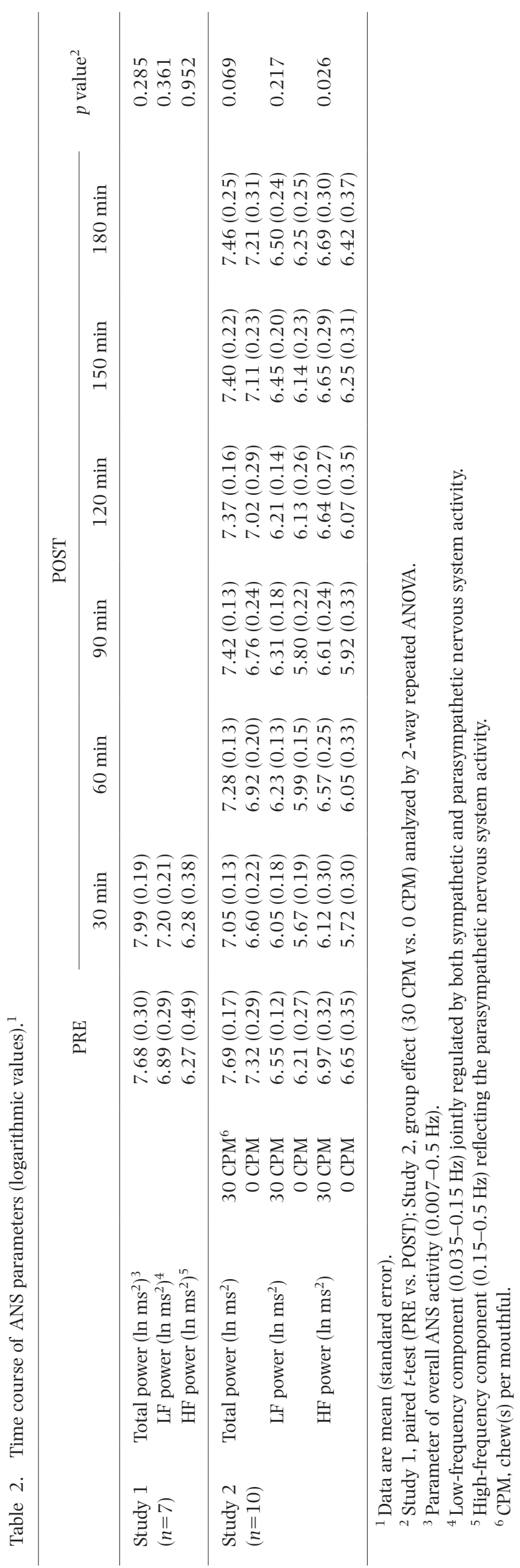


Table 3. Postprandial EE, carbohydrate and fat oxidation, and the TEM. ${ }^{1}$

\begin{tabular}{|c|c|c|c|c|}
\hline & $\begin{array}{c}\mathrm{EE} \\
(\mathrm{kJ} / 3 \mathrm{~h})\end{array}$ & $\begin{array}{l}\text { Carbohydrate oxidation } \\
\qquad(\mathrm{kJ} / 3 \mathrm{~h})\end{array}$ & $\begin{array}{l}\text { Fat oxidation } \\
\qquad(\mathrm{kJ} / 3 \mathrm{~h})\end{array}$ & $\begin{array}{l}\text { TEM }(\mathrm{kJ} / 3 \mathrm{~h}) \\
\% \text { energy intake }\end{array}$ \\
\hline $30 \mathrm{CPM}^{2}$ & $800.0(24.7)^{3}$ & $281.6(42.2)$ & $496.3(59.4)$ & $\begin{array}{c}134.2(15.5)^{4} \\
5.3(0.6)^{4}\end{array}$ \\
\hline 0 CPM & $740.0(14.6)$ & $355.0(53.0)$ & $371.2(61.4)$ & $\begin{array}{c}67.8(13.8) \\
2.7(0.5)\end{array}$ \\
\hline
\end{tabular}

${ }^{1}$ Data are mean (standard error). Total and net increases were calculated as the AUC. TEM, thermic effect of a meal.

${ }^{2} \mathrm{CPM}$, chew(s) per mouthful.

${ }^{3} p<0.05,{ }^{4} p<0.001$, paired $t$-test (30 CPM vs. 0 CPM).
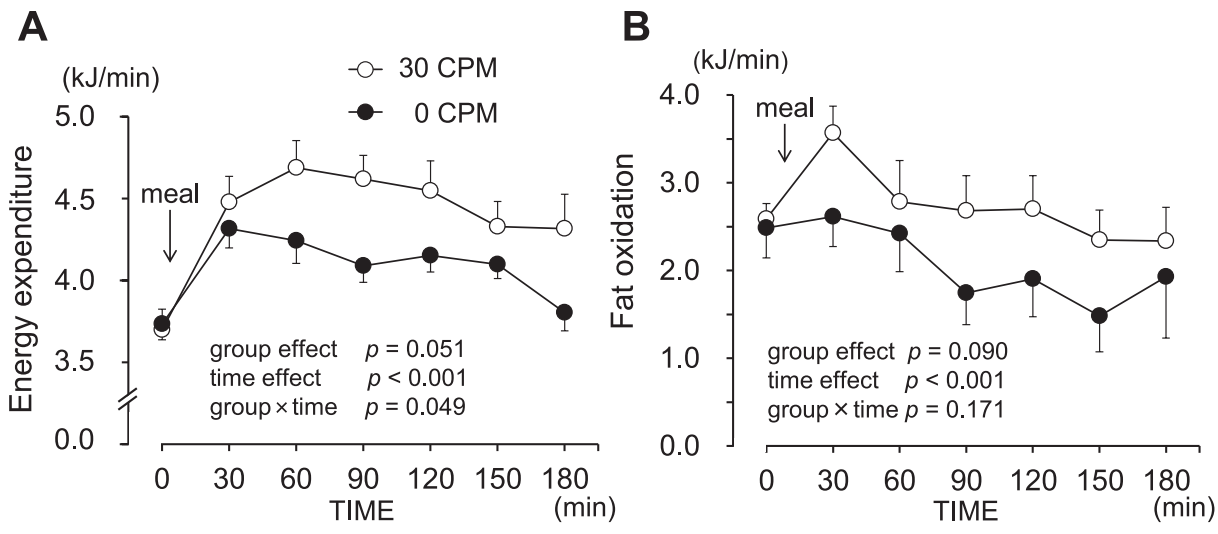

Fig. 2. Baseline and postprandial response for $\mathrm{EE}(\mathrm{A})$ and fat oxidation (B) during the $3 \mathrm{~h}$ after ingestion of a solid (30 CPM) or pureed meal (0 CPM). Each test meal was served immediately after the first measurement (time 0 min) as indicated by the arrow. Time effect, group effect and group $\times$ time were analyzed by 2 -way repeated ANOVA.

pared to baseline values $(p=0.003)$. The EE of mastication itself (mastication muscle activity) was calculated as baseline-adjusted AUC for EE, and was found to be $3.7 \pm 0.8 \mathrm{~kJ} / 20 \mathrm{~min}$.

There was no significant difference in fullness (PRE, $0.0 \pm 4.0 \mathrm{~mm}$; POST, $14.8 \pm 5.1 \mathrm{~mm} ; p=0.508$ ) or satiety ratings (PRE, $15.7 \pm 4.3 \mathrm{~mm}$; POST, $15.8 \pm 4.7 \mathrm{~mm}$; $p=0.224)$. The prospective food intake score was significantly decreased (PRE, $68.6 \pm 3.5 \mathrm{~mm} ; \quad$ POST, $68.1 \pm 3.6 \mathrm{~mm} ; p=0.024)$, and hunger ratings tended to decrease (PRE, $69.9 \pm 3.0 \mathrm{~mm}$; POST, $67.3 \pm 5.6 \mathrm{~mm}$; $p=0.096)$ after $20 \mathrm{~min}$ of mastication, although the gum sample did not contain any flavor or calories. No significant differences were found in any of the ANS activity parameters (Table 2 ).

Study 2

Masticatory efficacy on TEM and appetite sensations. Ten subjects consuming two test meals on separate days were included in the data analyses. No significant differences were found in the metabolic parameter values or in appetite ratings prior to eating the two meals (data not shown).

TEM: Postprandial EE, carbohydrate and fat oxidation, and the TEM are shown in Table 3. Significantly higher EE and TEM were observed after consuming the solid meal (30 CPM), compared to the pureed meal (0 CPM) (EE, $p=0.039$; TEM, $p<0.001$ ). Postprandial fat oxidation tended to be higher after consuming the solid meal than the pureed meal $(p=0.090)$. There was no significant difference in postprandial carbohydrate oxidation $(p=0.317)$.

The time course changes in EE and fat oxidation after consumption of the solid and pureed meals are shown in Fig. 2. Two-way repeated ANOVA showed a tendency of group effect in $\mathrm{EE}(p=0.051)$ and fat oxidation $(p=0.090)$, and significant interaction in EE (group $\times$ time, $p=0.049$ ), suggesting different time course changes in postprandial EE.

Appetite sensations: The time course changes in subjective appetite ratings, such as hunger, fullness, satiety, and prospective food intake, are shown in Fig. 3. Two-way repeated ANOVA revealed that there were no significant group (test meal) or group $\times$ time effects on hunger, fullness, or prospective food intake scores. However, significant group $(p=0.005)$ and group $\times$ time $(p=0.014)$ effects were observed for the time course of satiety, suggesting that mastication increases satiety.

Postprandial ANS activity response: The time course changes in ANS activity parameters are shown in Table 2. Two-way repeated ANOVA showed a tendency of group effect on total power, which reflects overall ANS activity $(p=0.069)$. In addition, there was a significant group effect observed on high-frequency (HF) power, which reflects parasympathetic activity $(p=0.026)$, suggesting higher parasympathetic activity during the postprandial period of the solid meal. There was no 

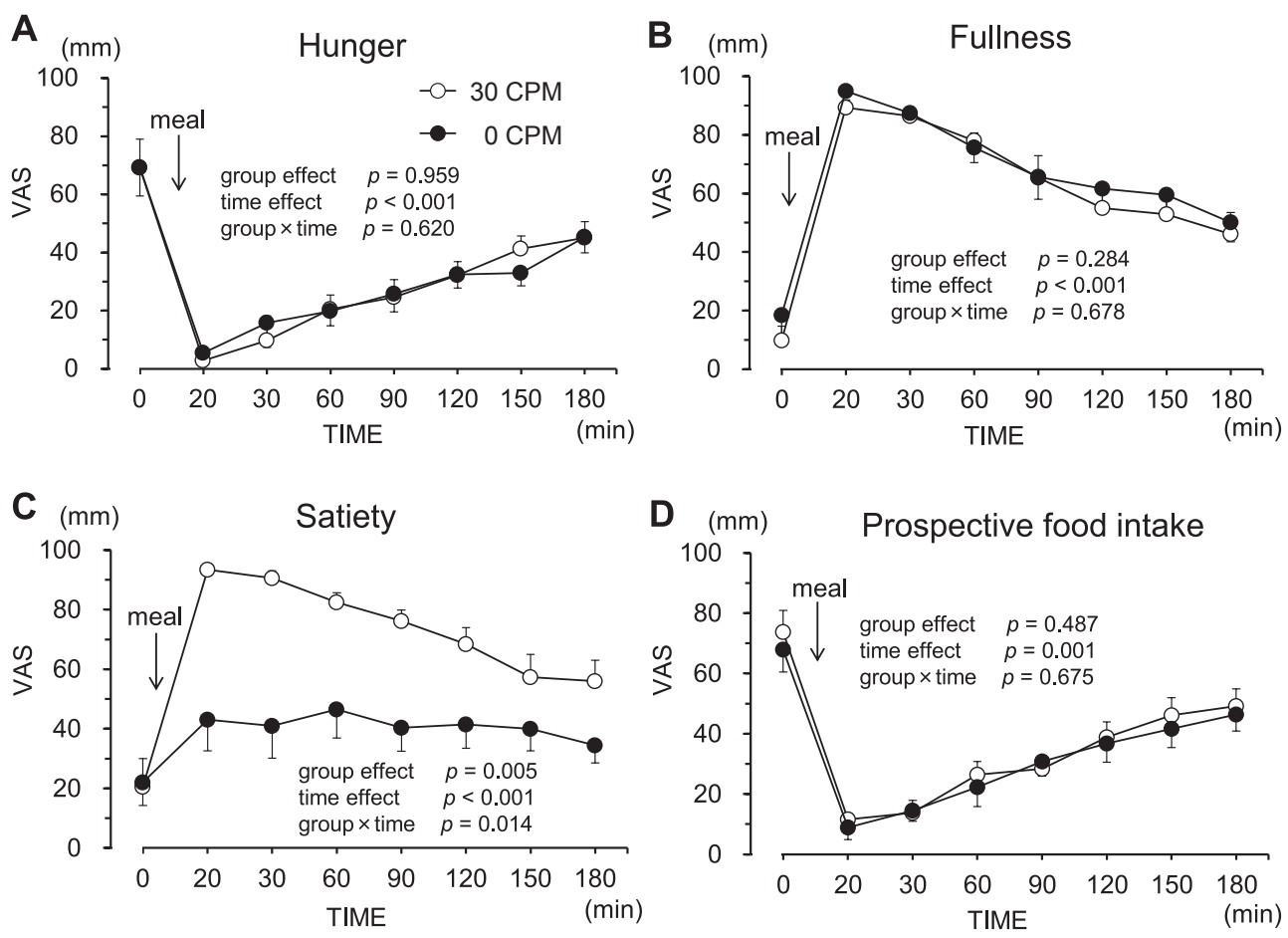

Fig. 3. Postprandial changes in subjective sensations of hunger (A), fullness (B), satiety (C), and prospective food intake (D) measured by visual analogue scales (VAS) during the $3 \mathrm{~h}$ after ingestion of a solid (30 CPM) or pureed meal (0 CPM). Each test meal was served immediately after the first measurement (time $0 \mathrm{~min}$ ) as indicated by the arrow. Time effect, group effect and group $\times$ time were analyzed by 2 -way repeated ANOVA.

significant group effect on low-frequency (LF) power, a parameter jointly regulated by both sympathetic and parasympathetic nervous system activity.

\section{DISCUSSION}

Our study presents three major findings. First, the TEM after solid meal consumption (30 CPM) was approximately twice as high as that after the pureed meal (0 CPM). Second, a higher feeling of satiety was observed after the solid meal (30 CPM) despite similar rating of postprandial feeling of fullness to that for the pureed meal (0 CPM). Third, the EE of mastication itself (masticatory muscle activity) was relatively small.

Mastication has been defined in a broad sense as the entire physiological process carried out in the oral and pharyngeal cavity while chewing food for swallowing and digestion (19). Therefore, the role of mastication functions not only for the grinding of food, but also to provide alimentary, oro-sensory information (smell, taste, and physical form) to the brain. Studies on humans have indicated that there is an important association between alimentary sensory stimulation and postprandial thermogenesis $(2,3,5)$. As reported by Brondel et al. (5), the TEM was approximately half $(30.3 \mathrm{~kJ} / 110 \mathrm{~min})$ when the test meal $(2,582 \mathrm{~kJ})$ was given as an infusion directly into the stomach when compared to the same meal ingested orally $(63.9 \mathrm{~kJ} / 110 \mathrm{~min})$. Moreover, the authors (5) also found that aliment sensory stimulation itself (sight, smell, and taste without swallowing) increased resting EE for the same period of time $(17.4 \mathrm{~kJ} / 110 \mathrm{~min})$, suggesting that both sensory and metabolic events may be involved in the early (cephalic) phase of postprandial thermogenesis. Similar results were reported by De Jonge et al. (3), indicating lower TEM after tube feeding $(1,621 \mathrm{~kJ})$ compared to oral feeding (1,638 kJ) (83 vs. $175 \mathrm{~kJ} / 6 \mathrm{~h}$ ). Interestingly, LeBlanc et al. (2) demonstrated that ingestion $(3,156$ kJ) with chewing (a solid meal) or without chewing (liquid meal) resulted in a marked difference in TEM response, suggesting the TEM, at least in the postprandial cephalic phase, may be influenced by sensory and cognitive stimulation. The present result regarding TEM is in good agreement with these previous reports $(2,3,5)$.

An animal study using dogs has demonstrated the role of ANS on both the cephalic and digestive phases of postprandial thermogenesis (20). Briefly, atropine (betaadrenergic blocker) inhibited postprandial thermogenesis during the cephalic (58\%) as well as the digestive phase (36\%). Moreover, propranorol (parasympathetic nerve blocker) plus atropine abolished thermogenesis completely during the digestive phase, suggesting both branches of the ANS exert a control on the TEM that occurs during the cephalic and digestive phases (20, 21). In the present study, a tendency toward greater overall ANS and higher parasympathetic nervous system activity was observed during the postprandial period after the solid meal, consistent with previous observations (21). We also observed that all autonomic parameters decreased after consumption of the meal compared to before the meal. Although changes in the autonomic balance after ingestion of water (22) or a meal $(23,24)$ have been reported previously, the mecha- 
nism of such autonomic response to ingestion of a meal is still unclear. A human study conducted by LeBlanc and Brondel (25) supported the role of ANS on the TEM. Briefly, a palatable meal $(2,971 \mathrm{~kJ})$ induced a markedly higher postprandial noradrenalin release together with greater TEM in the early phase compared to the same, non-palatable blended meal (70 vs. $45 \mathrm{~kJ} / 90 \mathrm{~min}$ ). This result raised the possibility that greater TEM after a solid meal with mastication (30 CPM) may be influenced by aliment and cognitive sensory stimulation during oral processes, at least during the early postprandial phase.

Focusing on postprandial appetite sensations, Li et al. (11) reported that after consumption of a test meal $(2,200 \mathrm{~kJ}$, pork pie) with $40 \mathrm{CPM}$ before swallowing, higher postprandial satiety as well as $12 \%$ less energy intake when consuming a meal ad libitum was observed in lean young men compared to consumption with 15 CPM. Similarly, Zhu et al. (12) proposed that the increase in chewing frequency (40 vs. 15 CPM) diminished postprandial appetite after the same test meal $(2,050 \mathrm{~kJ}$, pizza) in 18 - to 40 -y-old men. In previous studies using the ad libitum meal intake method, Smit et al. (26) reported that more chewing (35 vs. 15 CPM) reduced subsequent food intake in adults of various age and BMI; on the other hand, Zhu and Hollis (27) found age-related differences in the masticatory effect. Briefly, increase in chewing (150\% and $200 \%$ of the habitual amount of chewing) did not affect meal intake in older subjects, but a substantial reduction was observed in adults with various ages and BMIs. The increase in postprandial satiety with increased mastication observed in the present study is entirely consistent with previous reports $(11,12,28)$. These earlier reports suggest that increasing mastication reduces appetite ratings as well as increasing plasma concentration of satiety hormones for $3 \mathrm{~h}$ after ingestion. We observed higher postprandial satiety despite nearly identical hunger, fullness, and prospective food intake ratings, and it is uncertain why such a difference emerged. A meta-analysis reported by Flint et al. (29) suggested that insulin response may be a more powerful indicator of postprandial satiety in short-term appetite regulation in normal-weight subjects. Therefore, further study is needed to confirm the present results.

As for the EE of mastication itself, EE derived from masticatory muscle activity was estimated to be about $1 \mathrm{kcal}$. The resting metabolic rate (RMR), $3.5 \mathrm{~mL} / \mathrm{kg} /$ min, is characterized as the metabolic cost of resting quietly (30). The RMR of the present subjects was $3.5 \pm 0.1 \mathrm{~mL} / \mathrm{kg} / \mathrm{min}$ before gum chewing, indicating that our instrument for gas analysis was accurate and capable of tracking subtle changes in the subjects' gas flow and concentration. Regarding the impact of gum chewing on EE, Levine et al. (31) reported a greater mastication-induced thermogenesis (MIT, $11 \mathrm{kcal} / \mathrm{h}$ ) after 12-min non-nutritional gum chewing. The inconsistency between previous results (31) and our data may be due to two reasons: first, Levine et al. calculated postprandial thermogenesis for $1 \mathrm{~h}$ after chewing, but we estimated the EE derived from masticatory muscle activ- ity under chewing for $20 \mathrm{~min}$; second, Levine et al. used more gum $(8.4 \mathrm{~g})$ than we did $(1.0 \mathrm{~g})$. Further study was required to confirm our data. Some results have been reported in which gum chewing after lunch (32-34) led to a reduction in appetite sensations and subsequent snack intake. Considering the above reports and our results, the EE of mastication itself (masticatory muscle activity) may be relatively small, but mastication may have a greater metabolic effect on postprandial thermogenesis and appetite sensations.

The present study has some limitations. First, the sample size was small. Second, the different palatability between the two test meals may influence postprandial appetite and TEM. Finally, gut peptide secretion as well as insulin response are known to act as a stimulant on postprandial appetite sensations, but were not measured; therefore, the data should be interpreted carefully. Nevertheless, the present study demonstrates the effect of mastication on postprandial satiety and thermogenesis in humans. In conclusion, our results suggest that mastication increased TEM and satiety in young women. Chewing meals thoroughly may be a useful behavior for controlling appetite.

\section{Acknowledgments}

We are grateful to all participants for their cooperation. This work was partially supported by the Japanese Council for Science, Technology and Innovation, SIP (Project ID14533567), Technologies for Creating Nextgeneration Agriculture, Forestry and Fisheries (funding agency: Bio-oriented Technology Research Advancement Institution, NARO). The authors have no declared that no conflict of interest exists.

\section{REFERENCES}

1) Karl JP, Young AJ, Rood JC, Montain SJ. 2013. Independent and combined effects of eating rate and energy density on energy intake, appetite, and gut hormones. Obesity 21: E244-E252.

2) LeBlanc J, Cabanac M, Samson P. 1984. Reduced postprandial heat production with gavage as compared with meal feeding in human subjects. Am J Physiol 246: E95-E101.

3) De Jonge L, Agoues I, Garrel DR. 1991. Decreased thermogenic response to food with intragastric vs. oral feeding. Am J Physiol 260: E238-E242.

4) Sekhar RV, Shetty PS, Kurpad AV. 1998. Diet induced thermogenesis with oral \& intravenous feeding in chronically undernourished human subjects. Indian J Med Res 108: 265-271.

5) Brondel L, Fricker J, Fantino M. 1999. Postprandial thermogenesis and alimentary sensory stimulation in human subjects. Int J Obes Relat Metab Disord 23: 34-40.

6) Fujise T, Yoshimatsu H, Kurokawa M, Fukagawa K, Nakata M, Sakata T. 1993. Food consistency modulates eating volume and speed through brain histamine in rat. Brain Res Bull 32: 555-559.

7) Sakata T, Yoshimatsu H, Masaki T, Tsuda K. 2003. Antiobesity actions of mastication driven by histamine neurons in rats. Exp Biol Med (Maywood) 228: 1106-1110.

8) Oka K, Sakuarae A, Fujise T, Yoshimatsu H, Sakata T, Nakata M. 2003. Food texture differences affect energy 
metabolism in rats. J Dent Res 82: 491-494.

9) Smeets AJ, Westerterp-Plantenga MS. 2006. Oral exposure and sensory-specific satiety. Physiol Behav 89: 281-286.

10) Kokkinos A, le Roux CW, Alexiadou K, Tentolouris N, Vincent RP, Kyriaki D, Perrea D, Ghatei MA, Bloom SR, Katsilambros N. 2010. Eating slowly increases the postprandial response of the anorexigenic gut hormones, peptide YY and glucagon-like peptide-1. J Clin Endocrinol Metab 95: 333-337.

11) Li J, Zhang N, Hu L, Li Z, Li R, Li C, Wang S. 2011. Improvement in chewing activity reduces energy intake in one meal and modulates plasma gut hormone concentrations in obese and lean young Chinese men. Am J Clin Nutr 94: 709-716.

12) Zhu Y, Hsu WH, Hollis JH. 2013. Increasing the number of masticatory cycles is associated with reduced appetite and altered postprandial plasma concentrations of gut hormones, insulin and glucose. Br J Nutr 110: 384-390.

13) Nagai N, Sakane N, Tsuzaki K, Moritani T. 2011. UCP1 genetic polymorphism $(-3826 \mathrm{~A} / \mathrm{G})$ diminishes resting energy expenditure and thermoregulatory sympathetic nervous system activity in young females. Int $J$ Obes (Lond) 35: 1050-1055.

14) Lusk G. 1924. Animal calorimetry. Analysis of the oxidation of mixtures of carbohydrate and fat. J Biol Chem 59: $41-42$.

15) Task Force of the European Society of Cardiology and the North American Society of Pacing and Electrophysiology. 1996. Heart rate variability. Standard of measurements, physiological interpretation and clinical use. Circulation 93: 1043-1065.

16) Flint A, Raben A, Blundell JE, Astrup A. 2000. Reproducibility, power and validity of visual analogue scales in assessment of appetite sensations in single test meal studies. Int J Obes Relat Metab Disord 24: 38-48.

17) Nagai N, Hibi M, Yamaguchi T, Kameo Y, Kobayashi S, Katashima M. 2012. Development of the Japanese version of appetite sensations questionnaire using visual analogue scales (VAS), and assessment of its reproducibility and validity. J Jpn Soc Study Obes 18: 39-51 (in Japanese).

18) National Institute of Health and Nutrition. 2013. Dietary Reference Intakes for Japanese 2010, p 11. Daiichi Shuppan, Tokyo.

19) Kawamura Y. 1964. Recent concepts of the physiology of mastication. In: Advances in Oral Biology (Staple PH, ed), Vol 1, p 77-109. Academic Press, New York and London.

20) Diamond P, LeBlanc J. 1987. Role of autonomic nervous system in postprandial thermogenesis in dogs. Am J
Physiol 252: E719-E726.

21) LeBlanc J. 2000. Nutritional implications of cephalic phase thermogenic responses. Appetite 34: 214-216.

22) Routledge HC, Chowdhary S, Coote JH, Townsend JN. 2002. Cardiac vagal response to water ingestion in normal human subjects. Clin Sci (Lond) 103: 157-162.

23) Millis RM, Austin RE, Hatcher MD, Bond V, Goring KL. 2011. Metabolic energy correlates of heart rate variability spectral power associated with a 900-calorie challenge. J Nutr Metab 2011: 715361.

24) Nagai N, Sakane N, Ueno LM, Hamada T, Moritani T. 2003. The $-3826 \mathrm{~A} \rightarrow \mathrm{G}$ variant of the uncoupling protein-1 gene diminishes postprandial thermogenesis after a high fat meal in healthy boys. J Clin Endocrinol Metab 88: $5661-5667$.

25) LeBlanc J, Brondel L. 1985. Role of palatability on mealinduced thermogenesis in human subjects. Am J Physiol 248: E333-E336.

26) Smit HJ, Kemsley EK, Tapp HS, Henry CJ. 2011. Does prolonged chewing reduce food intake? Fletcherism revisited. Appetite 57: 295-298.

27) Zhu Y, Hollis JH. 2014. Chewing thoroughly reduces eating rate and postprandial food palatability but does not influence meal size in older adults. Physiol Behav 123: 62-66.

28) Cassady BA, Hollis JH, Fulford AD, Considine RV, Mattes RD. 2009. Mastication of almonds: effects of lipid bioaccessibility, appetite, and hormone response. Am J Clin Nutr 89: 794-800.

29) Flint A, Gregersen NT, Gluud LL, Møller BK, Raben A, Tetens I, Verdich C, Astrup A. 2007. Associations between postprandial insulin and blood glucose responses, appetite sensations and energy intake in normal weight and overweight individuals: a meta-analysis of test meal studies. Br J Nutr 98: 17-25.

30) Balke B. 1960. The effect of physical exercise on the metabolic potential, a crucial measure of physical fitness. In: Exercise and Fitness (Staley S, Cureton T, Huelster L, Barry AJ, eds), p 73-81. The Athletic Institute, Chicago.

31) Levine J, Baukol P, Pavlidis I. 1999. The energy expended in chewing gum. N Engl J Med 341: 2100.

32) Julis RA, Mattes RD. 2007. Influence of sweetened chewing gum on appetite, meal patterning and energy intake. Appetite 48: 167-175.

33) Hetherington MM, Boyland E. 2007. Short-term effects of chewing gum on snack intake and appetite. Appetite 48: 397-401.

34) Hetherington MM, Regan MF. 2011. Effects of chewing gum on short-term appetite regulation in moderately restrained eaters. Appetite 57: 475-482. 